\title{
STEM materials: a new frontier for an intelligent sustainable world
}

\author{
Pier F. Moretti ${ }^{*} \mathbb{D}$, Bartosz A. Grzybowski ${ }^{2,3}$, Vasileios Basios ${ }^{4}$, Elvira Fortunato ${ }^{5}$, Maria Suarez Diez ${ }^{6}$, Olga Speck ${ }^{7}$ \\ and Rodrigo Martins ${ }^{8}$
}

\begin{abstract}
Materials are addressed as possible enablers for solutions to many global societal challenges. A foresight exercise has been conducted to identify research paths to design, with a new approach, a generation of materials which can provide multi-functionalities. These material systems have been named "stem", in analogy to living cells where a base of primitive units can be designed and assembled for self-reacting to external inputs. These materials will embed a concept of "internet in things", where their processing capacity will enable the systems to interact with the environment and express diverse functionalities. Stem materials do not exist yet, but many clues from different theoretical and experimental results suggest they can be developed, and because living organisms exist. This article aims at launching this new approach and promoting the structuring of a multi-disciplinary community to fill the research gaps.
\end{abstract}

Keywords: Foresight, Internet in things, Multi-functional materials, Adaptive/living materials, Sustainability, Interfaces, Meaning of dynamics

Materials able to perform different functions and to respond to external inputs will become increasingly important, in particular for their contribution in tackling global challenges.

"Stem Materials" refer to a new generation of material systems which can impact in this regard.

The adjective "stem" has been originally attributed to living cells that can differentiate into other types of cells. By analogy, "stem" addresses here the use of blocks of non-specialized materials capable of change allowing them to adapt to specific requirements.

Global challenges, such as food, climate, energy, water and health, address the need of complex systems in an interconnection of diverse variables, sectors and stakeholders. In this context, research towards innovative systems enabling commodities of the future and boosting prosperity and well-being of citizens in agreement with the UN Sustainable Development Goals is essential.

Internet of Things will enable many solutions to many future global challenges, and it will definitely permeate

\footnotetext{
*Correspondence: pierfrancesco.moretti@cnr.it

${ }^{1}$ National Research Council, P.le A. Moro 7, Rome, Italy

Full list of author information is available at the end of the article
}

our future. In this context, digitalization, artificial intelligence and cyber security are considered the main drivers, and actors too. Indeed, the political and industrial attention seems to be focused on the role of internet, and less on that of the things. Reducing the risk of asymmetric shocks between the deluge of data and the appropriateness of controlled products, ask future routes to concentrate on "safety by design", and hopefully to simplify the complexity of the interconnected agents involved in the socio-economic-environmental system. The concept of Stem materials, which will be described shortly, will embed a new generation of human-synthesized systems, intelligent and able to feel in and react to the environment where they are but independent of internet, so allowing a complementary pathway toward sustainable solutions.

In this scenario, materials play a fundamental role, both as enablers for a variety of solutions and as key tools to reach the same. Indeed, increasing concerns about (critical) raw materials and sustainable use of natural resources are already fostering innovative processes in framing materials within the concept of circular economy [1] in terms of closed-loop materials and energy cycles, and within nature-based solutions to societal challenges. 


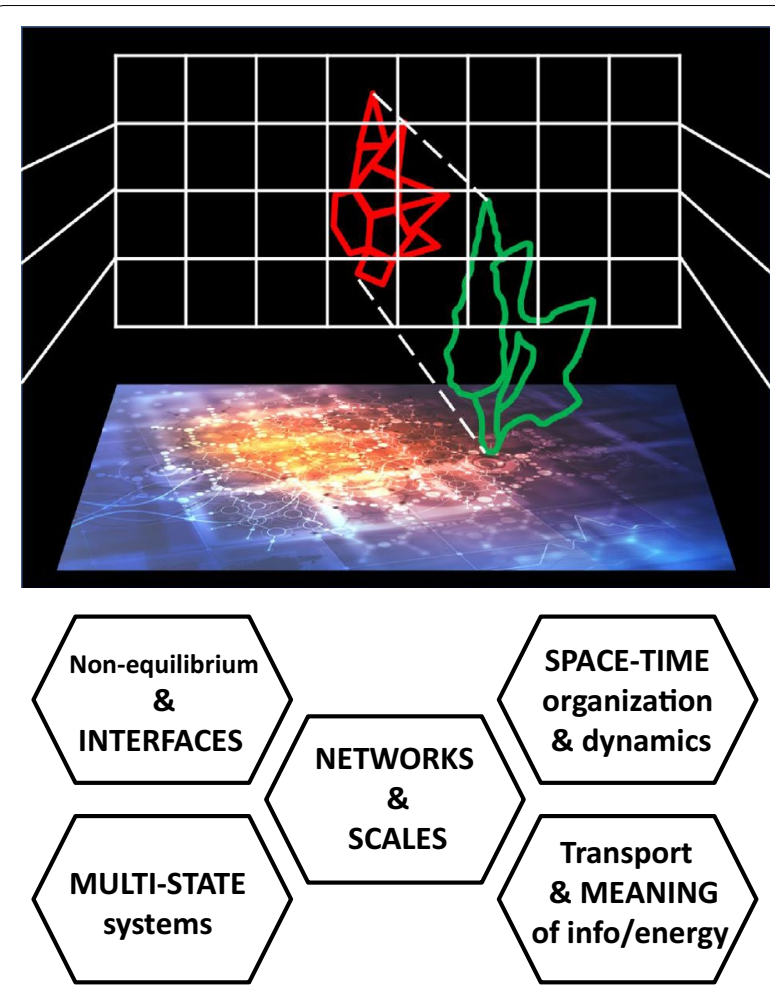

Fig. 1 The concept of Stem Materials. (Top) A fiction vision of Stem Materials, which aim at mimicking living organisms (the marine gastropod mollusk "Aporrhais pespelecani" is taken as an example of adaptation to environmental changes), designed and assembled from a base of primitive units for self-reacting to external inputs. The development of Stem Materials' concept asks for contributions from a wide variety of scientific disciplines and communities, in a complex integration of quantum mechanics and chemical synthetic biology, chemistry and genetics, engineering and manufacturing, in a truly and inter-disciplinary and multi-disciplinary approach. (Bottom) The main aspects which have to be investigated in order to achieve Stem Materials, as identified by the experts involved in the foresight exercise which launched the concept of Stem Materials

This renewed approach to materials aims at providing solutions that are inspired or supported by nature, which provide sustainable, cost-effective, multi-purpose, flexible and simpler alternatives for various objectives [2].

Moreover, answering the demands of societal challenges requires complex approaches but ultimately straightforward simpler solutions for systems and products, ensuring so both cost effectiveness and sustainability in terms of energy and environment.

In this context, multi-functional materials will definitely play a fundamental role in additive production to the extent that they are designed and structured to perform and adapt, without any additional devices (any electronics to answer to stimulus ought to be really embodied in their structure).
In nature, living organisms consist of a limited number of primary components and chemical bonds [3, 4], organized into complex systems capable of evolution and adaptation to diversified and changing environmental conditions often realized by materials systems. Synthetic materials are very rarely adaptable, and often require a large number of components to achieve high performances in specific functions. A comparison between living materials in organisms and synthetic materials in technical constructions shows that even the concepts of their respective life-cycles are largely different: the first renewing themselves in a continuous interaction with the environment, the latter mainly preserving themselves from alterations. Controlling self-organization, self replication and resilience versus adaptability is the main challenge at this point.

Although design and production of such systems has already started (sometimes referred as 4D-materials for their capacity to change properties with time) and advances in responsive materials have been notable [59], reversing the current manufacturing processes and introducing materials able to perform different sensing and actuation functions depending on external environmental conditions, is still a challenge. The same considerations concern the use of smart interfaces to respond to human needs.

These intelligent materials or even materials systems should be adaptable in any context and condition, and possibly consist of primitive units constituting a basis in a functional vector space (already alluded to as a materials' genetic code [10]), containing the minimal and sufficient number of components to perform some basic functions, as mirroring principles of live-cell modeling [11]. The combinations of these units should respond to specific requests of multi-functionality and adaptability, thanks to their multi-state and multi-level organization.

Several leading researchers from different disciplines recently met in the context of a foresight exercise [12] and coined the term "Stem Materials" to refer to this new generation of materials systems.

The challenge will be therefore to understand if there is the possibility to introduce a general and breakthrough mathematical framework for primitive units and their combinations, and/or an accurate and flexible empirical framework to satisfy any request for multi-functionality and multi-performance of materials (see a schematic representation of the concept of Stem Materials in Fig. 1).

The stem materials so will clear impact in the challenges we need to overcome for a better comfort and welfare of humankind. Such a challenge asks for contributions from a wide variety of scientific disciplines and communities, from quantum mechanics to chemical synthetic biology, from chemistry to genetics, from 
engineering to bio-mimetics and medicine, in a truly and inter-disciplinary and multi-disciplinary approach. This is clearly a challenging mission with potentially transformative impacts for our future.

Researchers present at the Foresight meetings identified some most relevant aspects to focus on, including the roles of interfaces and space-time dynamics of energy/information. The capability to manage multi-state systems/networks, which are non-local, present in various space-time scales, and dependent on the history of the process, seems to be the crucial aspect in designing [13] and inducing stem materials to be reversible in their functionalities.

In this regard, understanding reversibility and context dependency embedded in the concept of the stem materials can lead to wider-context breakthroughs in implementing sustainability and circular economy.

Indeed, stem materials will serve as drivers for the efficient use of resources because, unlike conventional materials, owing to their inherent potential for adaptability and change, no new materials need to be used under changing conditions or in various applications, besides reducing the complexity of the systems where it will be used. Thus, in the context of sustainable development, stem materials will lead to a paradigm shift, particularly in resource preservation and generally in understanding of nature-inspired intelligent solutions.

\section{Acknowledgements}

We thank Ezio Andreta, Giorgio Einaudi and Ruggero Casacchia for their support in structuring the Foresight Project. We also thank all the experts who participated to the many workshops and meetings, contributing to the discussions

\section{Authors' contributions}

PFM contributed to the conception and coordination of the Stem Materials' group, and drafted the article. RM and EF contributed in drafting and revising the article. BAG, VB, MSD and OS contributed to revise it. All authors read and approved the final manuscript.

\section{Funding}

The activities within the S\&T Foresight Project have been funded by the National Research Council of Italy. The funding body has not played any role in the design of the study and collection, analysis, and interpretation of data and in writing the manuscript. In turn, all these aspects were implemented by the authors within the mentioned Project.

\section{Availability of data and materials}

Findings reported in this article are based on discussion and personal contributions only. No data or material were used and are available for sharing.

\section{Competing interests}

The authors declare that they have no competing interests.

\section{Author details}

${ }^{1}$ National Research Council, P.le A. Moro 7, Rome, Italy. ${ }^{2}$ Institute of Organic Chemistry, Polish Academy of Sciences, ul. M. Kasprzaka 44/52, Warsaw, Poland. ${ }^{3}$ IBS Center for Soft and Living Matter and Department of Chemistry, UNIST, Ulju-gun, Ulsan, South Korea. ${ }^{4}$ Interdisciplinary Centre for Nonlinear Phenomena and Complex Systems, Statistical Physics and Complex Systems Department, Université Libre de Bruxelles, Avenue Franklin Roosevelt 50, Brussels, Belgium. ${ }^{5}$ Institute for Nanostructures, Nanomodelling and Nanofabrication, i3N and of the Centre of Research in Materials (CENIMAT) of Faculty of Science and Technology of Nova, University of Lisbon, Quinta da Torre, 2829-516 Caparica, Portugal. ${ }^{6}$ Laboratory of Systems and Synthetic Biology, Wageningen University and Research, Droevendaalsesteeg 4, Wageningen, The Netherlands. ${ }^{7}$ Plant Biomechanics Group and Botanic Garden, University of Freiburg and Center for Interactive Materials and Bioinspired Technologie, Fahnenbergplatz, Freiburg im Breisgau, Germany. ${ }^{8}$ Materials Science Department of Faculty of Science and Technology of Nova, University of Lisbon and European Academy of Sciences, Quinta da Torre, 2829-516 Caparica, Portugal.

Received: 19 May 2019 Accepted: 31 October 2019 Published online: 20 November 2019

\section{References}

1. Stahel WR. The circular economy. Nature. 2016:531:435-8.

2. European Commission: Nature based solutions. 2019. https://ec.europ a.eu/research/environment/index.cfm?pg=nbs. Accessed 6 May 2019.

3. Alberts B, Johnson A, Lewis J, Raff M, Roberts K, Walter P. Molecular biology of the cell. New York: Garland Science; 2002.

4. Vladilo G, Hassanali A. Hydrogen bonds and life in the universe. Life. 2018;8:1.

5. Warren SC, Guney-Altay O, Grzybowski BA. Responsive and non-equilibrium materials. J Phys Chem Lett. 2012;3:2103-11.

6. Z Zhang XA, Yu S, Xu B, Li M, Peng Z, Wang Y, Deng S, Wu Z, Ouyang $M$, Wang $Y$. Dynamic gating of infrared radiation in a textile. Science. 2019:363:619-23.

7. Mann S. Self-assembly and transformation of hybrid nano-objects and nanostructures under equilibrium and non-equilibrium conditions. Nat Mater. 2009;8:781-92.

8. Tibbits S. Active matter. Cambridge MA: The MIT Press; 2017.

9. Mayue S, Jinxin Z, Haotian C, Mengdi H, Smitha SA, Zongmin S, Bo M, Xiaoliang C, Haixia Z. Self-powered analogue smart skin. ACS Nano. 2016;104:4083-91.

10. Lejaeghere $\mathrm{K}$, et al. Reproducibility in density functional theory calculations of solids. Science. 2016. https://doi.org/10.1126/science.aad3000.

11. Luisi PL, Stano P. Synthetic biology: minimal cell mimicry. Nat Chem. 2011:3:755-6.

12. National Research Council of Italy: Foresight Working group Materials. 2018. http://www.foresight.cnr.it/working-groups/wg-materials. Accessed 6 May 2019

13. Newman M. The power of design. Nature. 2000;405:412-3.

\section{Publisher's Note}

Springer Nature remains neutral with regard to jurisdictional claims in published maps and institutional affiliations. 